\title{
Reflectance Spectrum of a Color Blue Generated by Curved Multilayer and Iridescent of the Elytron of the Calidea Signata Bug
}

\author{
Issaka Ouedraogo, PhD \\ Serge Wendsida Igo, PhD
}

Research Institute of Applied Science and Technology, Ouagadougou, Burkina Faso

Priscilla Simonis, PhD

Research Center in Physics of Matter and Radiation, Namur University, Belgium

Alioune Ouedraogo, Professor

Ouagadougou University- UFR-SEA Ouagadougou, Burkina Faso

Belkacem Zeghmati, Professor

Mathematics and Physics Laboratory, University of Perpignan, France

doi: 10.19044/esj.2016.v12n36p1 URL:http://dx.doi.org/10.19044/esj.2016.v12n36p1

\begin{abstract}
This paper focuses on the study of the origins of blue iridescent reflections of the elytron of Calidea Signata, of the pentatomidae family which is commonly known as bug. Indeed, we use the spectrophotometry measurements, scanning electron microscopy characterization technique, and a ray tracing code program combined with the transfer matrices method, to explain the structure which is responsible for the color of the blue reflections and the iridescent effect. Thus, we noticed that the color of the bug as a result of two peaks. The first and main peak is located at $485.5 \mathrm{~nm}$, corresponding to the blue color. The second peak, is less intense at $525 \mathrm{~nm}$, corresponding to the green-blue color. The numerical reproduction of the reflectance spectrum gives $510.25 \mathrm{~nm}$ for the main peak, and $539.5 \mathrm{~nm}$ for the secondary peak. These results confirm that the multilayer structure is responsible for the blue color of the Calidea Signata bug. Lastly, the curve of the multilayer is responsible for the iridescence.
\end{abstract}

Keywords: Blue, Reflectance, Multilayer, Iridescence. 


\section{Introduction}

From nature, we can find the wings of insect producing a great variety of colors due to reflection. This is common in living organisms. For example, the Litoria cauerulea from the Southern New Guinea produces a color mixture in reflection, going from green to blue (Fox, 1976). In this study, the specimen has a rare mixture of color, from brown to blue and green. However, this is a bug, called Calidea signata from the pentatomidae family. It is from the Podopinae sub-family, of the Hemiptera order. The back face of the bug is characterized by two pairs of membranous wings protected by elytron (solid wings). This elytron has a non-metallic iridescent color ranging from blue to green (Balint et al., 2005; Berthier, 2007). Generally, this color is due to its multilayers. Therefore, a multiscale approach is needed to explain the iridescence (Parker, 2005).

The color of the Calidea signata plays an important role in the insect's life in its environment. The color shows the family membership and it helps in ensuring the species' reproduction. Therefore, an insect with many colors is genetically better fit for its race. The color enables one differentiate between the male and female. The objective of the male is to attract the female with its rich genetic. Thanks, therefore, to its colors. On the other hand, the female is more discreet, and less colored, so as to escape predators. Calidea signata is a phytohagous insect.

Figure. 1 shows the bug called Calidea signata, which lives in tropical climate in West Africa. Consequently, the specimen was identified for the first time in 1939 (Paul Freeman). Their number is estimated at 550 species (Schuh \& Slater, 1995; Vigneron and al., 2010; ICI, 1931). The specimen is from the collection of the Natural History Museum of Burkina Faso National Centre for Scientific Research and Technology (CNRST). We noticed that the long storage period in the museum has not altered the elytron color of the Calidea signata. This, however, is a further evidence of a structural coloring, which is contrary to pigment coloring. Indeed, the pigment coloring is sensitive to ultraviolet. This makes it very degradable during storage. We use spectrophotometer, scanning electron microscope (SEM) techniques, and a numerical method based on multi-scale approach to explain the origin of the blue reflections of the elytron, especially the iridescence of the bug.

However, these multilayers are interesting in trapping nanometer scale light. Today, with the Nano-photonic group deposition methods, they can make new materials. 


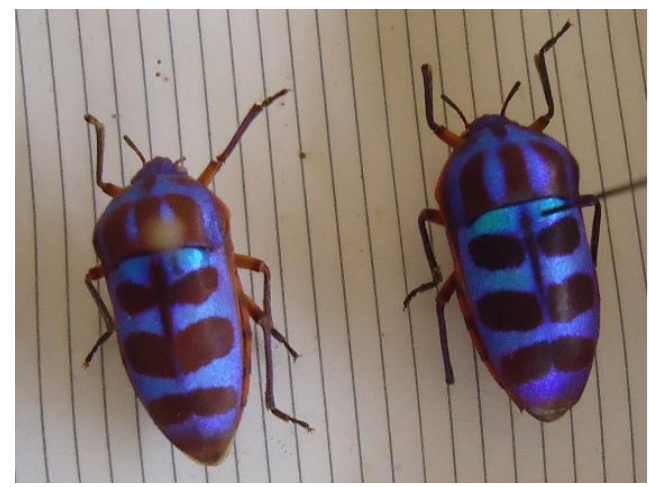

Figure 1. The Calidea signata (Scutelleridae) specimen

\section{Spectrum Measurement}

A dry sample of the dorsal area of Calidea Signata male of size $0.5 \mathrm{~cm}$ $\mathrm{x} 0.5 \mathrm{~cm}$ was measured using a spectrophotometer (Avaspec-2048). In the case of Avaspec-2048, the reference is a white diffuser and the material of reflectance spectrum is greater than $98 \%$ in the visible. Here, we will focus on the unique angle $\theta=15^{\circ}$. This is because the other angles of $30^{\circ}, 45^{\circ}, 60^{\circ}$, and $75^{\circ}$ provides no additional information.

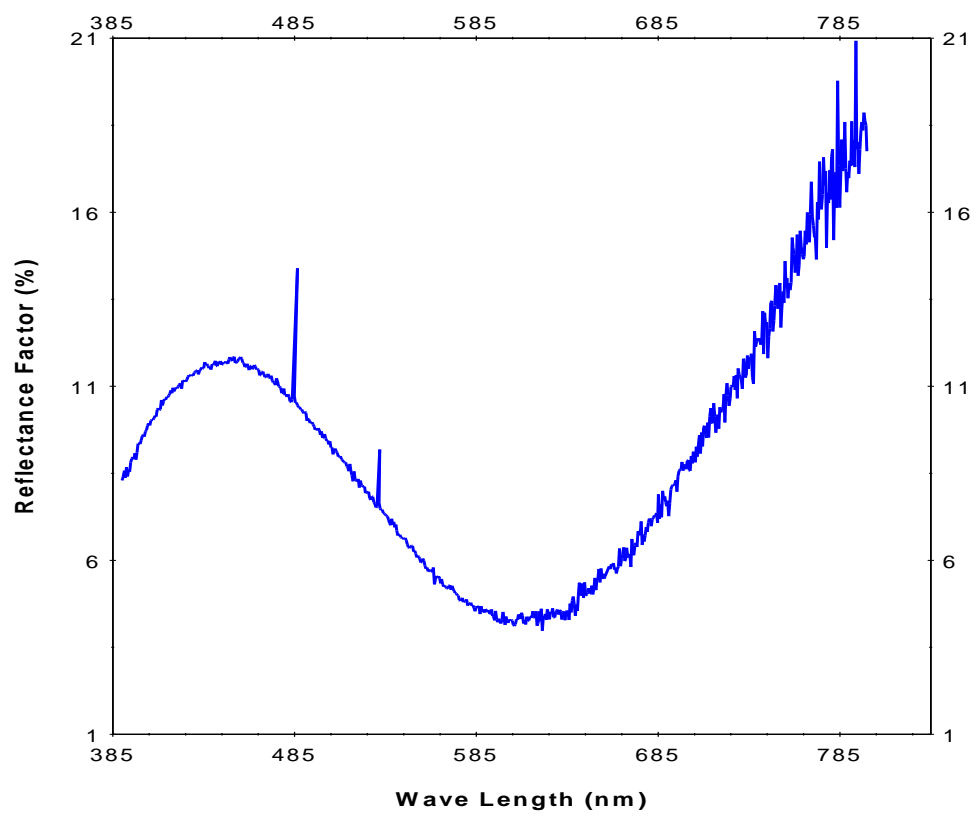

Figure 2. Reflectance spectrum of elytron of the Calidaa Signata, measured at normal incidence of $\theta=15^{\circ}$.

The reflectance spectrum of Figure 2 shows a main peak at $485.5 \mathrm{~nm}$, a blue one. The second peak is at $525 \mathrm{~nm}$, corresponding to the blue-green color. In addition, the bug color is made of both two peaks (Kelly, 1976; $\mathrm{Zi}$ and, 2003). 


\section{Nano-morphology Observation with Scanning Electron Microscopy}

The observation of the samples of the same size such as the dorsal elytron spectrophotometer of the Calidea signata with SEM will enable us to find the possible origin of the blue and bluish green colors. Indeed, we take a piece of the elytron or cuticle of the bug. Then, this piece is broken into two parts in liquid nitrogen at very low temperature. This is done to maximize the risk of a complete break. Also, we use JOEL SEM 7500F type for observation.

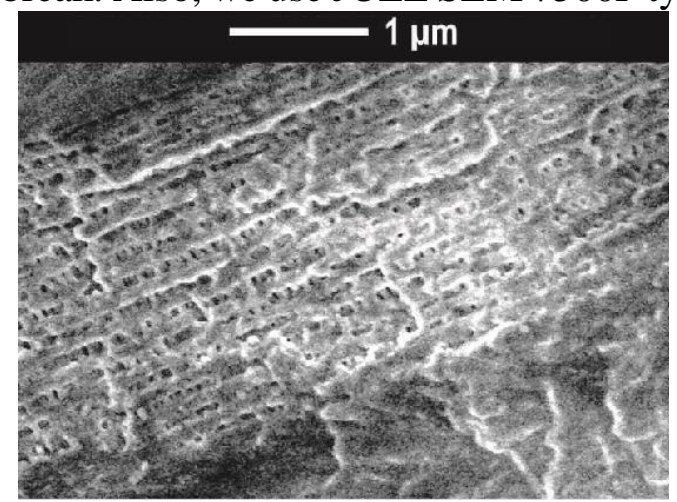

Figure 3. Image scanning electron microscope view of the cuticle (elytron) cross section of the calidea signata. Magnification x 45000 JOEL7500 F.

Figure 3 shows one of the obtained SEM images from the cross section of the calidea signata. Magnification x 45000 JOEL7500 F.

Thus, we can see that it is multilayer. It is made of chitin and chitinempty area, of nearly fifteen bilayers. Thanks to SEM images. The period is estimated at $172 \mathrm{~nm}$ for the whole multilayer. The multilayer can be considered as a homogeneous material with an average refractive index $\bar{n}=$ 1.5 with $m=1$ ( $m$ is an integer). Thus, we can calculate the wavelength which was reflected with the following expression in a system called Bragg mirror (Prendry \& Kinnon, 2005):

$$
\lambda=\frac{2 \mathrm{a} \sqrt{\overline{\mathrm{n}}^{2}-\sin \theta^{2}}}{\mathrm{~m}}
$$

The numerical application (1) gives, $\lambda=508.25 \mathrm{~nm}$, when compared with the length of the spectrum $(485.5 \mathrm{~nm})$. We can see that they fairly align, despite the simplicity of the method used, and assuming the multilayer as a homogeneous medium has an equivalent refractive index. Therefore, we noticed that the multilayer is at the origin of the blue color.

$$
\lambda=\frac{2 \mathrm{a} \sqrt{\overline{\mathrm{n}}^{2}-\sin ^{2} \varphi}}{\mathrm{m}}
$$

The position of the secondary peak, due to the double reflection, can be found by adapting the formula (1) to (2) of the dominant wavelength, $\lambda$ $=535.5 \mathrm{~nm}$. The secondary peak is due to the geometric shape of the elytron 
of Calidae signata. Indeed, the back faces of the elytron were not smooth, but contains small spherical cavities. The angle $\theta=15^{\circ}$ corresponds to an angle $\varphi=37.5^{\circ}$ in the curve. The relationship between the local incidence angle $\varphi$ and the overall incidence angle $\theta$ is given by the following equation $\varphi=\frac{\pi}{4}-$ $\frac{\theta}{2}$.

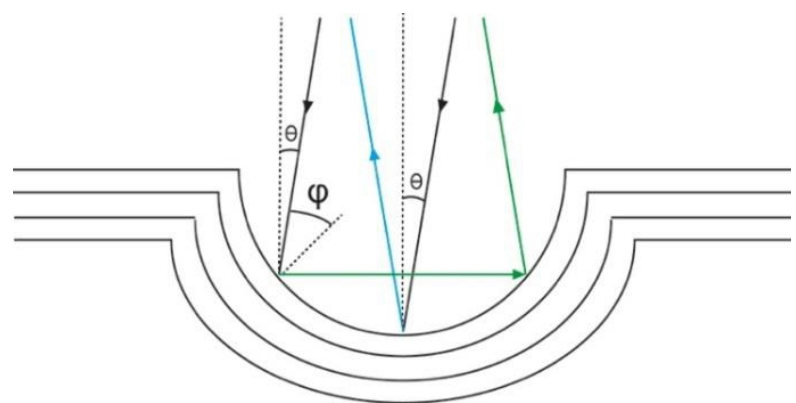

Figure 4. Geometrical Schematic of cross section of a spherical cavity: rays reflections process using a curved iridescent. The description of this mechanism requires a multiscale approach.

The interpretation of the origin of the color is as follow: the incident rays, according to the area encountered. If the ray reflects on an area located between two spherical cavities, the multilayer is flat, while the color observed at normal incidence is blue (Figure 4). However, if the ray reflects on a cavity, two cases can be observed. Either the ray reflects on the center of the cavity, or in this case, the multilayer can be considered as flat or slightly curved, and the reflected ray is blue. On the other hand, the ray strikes the edge of the cavity. It then reflects firstly on the opposite side, and then reflects secondly towards the outside. The color of the reflected ray is green-bluish. As shown in Figure 4, the spherical cavities cause the double reflection of one part of incident rays, i.e. those that reflect on the curved edges of these cavities. An interesting property therefore results from this, iridescence.

\section{Modeling of the Curved Iridescent Multilayer Comprising Spherical Cavities}

To model the measured reflectance spectrum and the structure observed with SEM (multilayer with spherical cavities) on elytron of Calidea signata, we use the ray-tracing program combined with the transfer matrix method. Thus, a multiscale approach is necessary to describe the code formalism which makes it possible to couple coherent reflections with incoherent diffusion (Tian et al., 2007; Gohy \& Melinte, 2009; Huang et al., 2008).

The modeling will be used to define the objects used in the code. Then, we will describe the propagation of light with the ray-tracing method. Since 
the approximate number of layers of the multilayer is known, modeling can be described as infinite and one-dimensional photonic crystal. The elytron of Calidea Signata presents an iridescent and curved surface on the edges. This curvature causes multiple reflections of the incident beams. Therefore, this leads to an inconsistent mixture of the various wavelengths. Also, the spectral content of light diffused is easily accessible. This is based on the fact that light diffusion in structured materials is known with Maxwell's equations for diffraction on a smaller scale than that of the wavelength, and with geometric optics for large scale diffusion. Consequently, the difficulty is on how to combine both systems at different scales into a single system.

\section{Describing Ray Tracing Program}

Four objects which enable the description of the ray tracing method code are: light ray, surface element, source element, and detector element. Thus, each object is defined below.

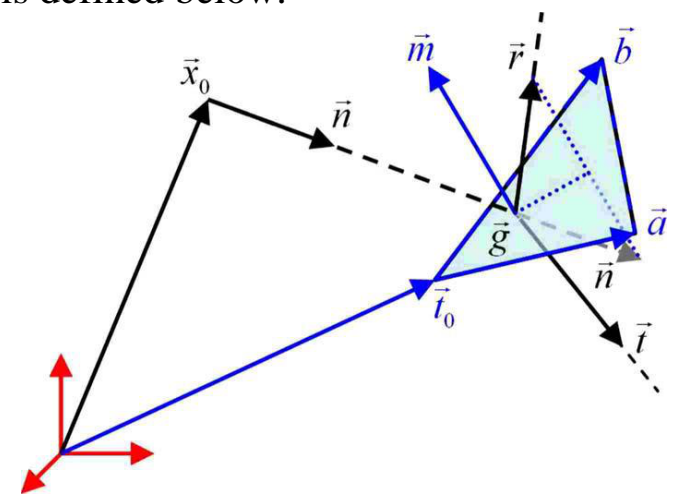

Figure 5. Geometrical representation of ray tracing procedure (line color)

'The light beam' is an object (Figure 5) which has an origin $\overrightarrow{x_{0}}$ and a propagation direction $\vec{n}$, a standardized vector. It also includes a third vector $\vec{p}$, also standardized, linked to the linear polarization, and forced to be perpendicular to the propagation direction (not shown in the diagram). Lastly, they are also associated with the first object, a specific wavelength $\lambda$, and intensity $i_{0}$.

'Surface element' is a triangle (Figure 5) defined by the two vectors $\vec{a}$ and $\vec{b}$, each of these vectors originating from $\overrightarrow{t_{0}}$. At the conceptual level, this surface has an internal face and an external one. The standardized vector $\vec{m}$, perpendicular to vectors $\vec{a}$ and $\vec{b}$, shows the external direction. To this, surface element are added parameters related to the multilayer, $n_{i}$ gives the number of layers, $d_{i}\left(i=1, \ldots n_{i}\right)$ represents the thicknesses of layers, and $\varepsilon_{i}(i=$ $\left.1, \ldots, n_{i}\right)$ are the respective dielectric constants of the layers. 
'The source element' is also a triangle (Figure 5) with the same geometric parameters, $\vec{a}, \vec{b}, \overrightarrow{t_{0}}$ and $\vec{m}$. No multilayer is associated with it. Thus, this is contrary to the surface element. However, a new vector, $\vec{f}$ is defined. This vector indicates the beam propagation direction. Polarization is also taken into account, by two orthogonal vectors and the direction of propagation. Each ray emitted by the source is associated with a polarization vector, random linear combination of these two vectors, Therefore, any possible polarization can be taken into account. Thus, for a linear polarization, one of the vectors is set to zero. In the case of a non-polarized light, each vector has an identical standard.

'The detector element' is still a triangle (Figure 5) which is always described by the parameters, $\vec{a}, \vec{b}, \overrightarrow{t_{0}}$ and $\vec{m}$. The particularity of this object is that it includes a multi-channel system consisting of a set of $n_{\lambda}$ intensities, initially null, which covers a range of wavelengths from $\lambda_{\min }$ to $\lambda_{\max }$. When the beam reflects on the detector, this latter is removed. Also, its intensity is added to the appropriate channel, that is related to the specific wavelength of the ray.

\section{Ray Tracing Procedure}

Since objects are defined, we need to explain the ray-tracing process. First, the structure to be analyzed is cut into triangles which constitute the surface elements. Similarly, light source and the detectors are defined by a set of triangles, i.e. source element and detectors, respectively. A ray list, initially empty, is prepared. Then, a first beam, produced from a random point in a source element which is also chosen randomly, is sent. This first beam is stored in the list of beam. It has its own characteristics. Its intensity and its polarization were determined in advance, and it is closer to the experimental conditions. The beam is sent in a direction of the focus of the source. Once these steps are completed, the ray-tracing process is therefore launched.

This module examines the beam at the top of the list of beams mentioned above. It then analyzes whether this beam does not go across something (in this case, it is then deleted) or hit an obstacle. This obstacle, which may be either surface element or a detector element, is anyway a triangle. To see if a beam hits the surface of a triangle, it is important to observe if the contact point $\vec{g}=\overrightarrow{x_{0}}+t \vec{n}$ is consistent with a point in the plane of the triangle $\vec{p}=\overrightarrow{t_{0}}+u \vec{a}+v \vec{b}$. We need to solve the system of the three linear equations $\vec{a}+v \vec{b}-t \vec{n}=\overrightarrow{x_{0}}-\overrightarrow{t_{0}}$ with three unknowns $u$, $v$ and $t$. To have a contact, we need to meet the following four conditions: $0 \leq u<1,0 \leq$ $v<1, \quad u+v<1, \quad t \geq 0$. These four equations ensure that the Point is located within the triangle. If it comes into contact with a triangle, it determines what type it is this: a surface element or a detector element. In the 
first case, the beam is replaced, in the list. This is done by the reflected and sent beam, with their own intensities and polarizations. It should be noted that at this stage, if the beam intensity, whatever it is, is below the pre-established level, the beam is removed from the list. In the second case, the beam is removed from the list, when its intensity and polarization are stored in the appropriate channel. Subsequently, this procedure is repeated until there are no more beams in the list. A new beam is then launched from the source, and the process is resumed.

\section{Parameters of Reflected and Sent Beams}

If a beam $\left[\overrightarrow{x_{0}}, \vec{n}\right]$ meets a surface element $\left[\overrightarrow{t_{0}}, \vec{a}, \vec{b}, \vec{m}\right]$ at the point of contact $\vec{g}$, this beam must be replaced by its reflected and transmitted successors. The reflected beam is defined by $[\vec{g}, \vec{r}]$, or the point of contact $\vec{g}$ is defined as its origin, and or:

$$
\vec{r}=\vec{n}-2(\vec{n} \cdot \vec{m}) \vec{m}
$$

Here, $\vec{n}$ and $\vec{m}$ is defined standardized vectors. It should be noted that the vector giving the direction of propagation, $\vec{r}$, must be standardized after evaluation. The origin of the transmitted beam $[\vec{g}, \vec{t}]$ is also defined at the point of contact $\vec{g}$. Concerning the direction of propagation, we must take the refraction into account. Thus, the direction of propagation is given by the following relation:

$$
\vec{t}=\cos \theta_{\text {out }} \vec{n}+\sin \theta_{\text {out }} \vec{u}
$$

Where $\vec{u}$ defines the plane of incidence:

$$
\vec{u}=\frac{\vec{n}-(\vec{n} \cdot \vec{m}) \vec{m}}{|\vec{n}-(\vec{n} \cdot \vec{m}) \vec{m}|}
$$

Furthermore, what remains is to determine the expressions $\sin \theta_{\text {out }}$ and $\theta_{\text {out }}$. The angle of incidence of $\theta_{\text {in }}$ is given by the following relationship:

$$
\sin \theta_{\text {in }}=|\vec{n} \cdot \vec{m}|
$$

Therefore, according to Snell's law, the transmitted ray emerges at an angle $\theta_{\text {out }}$ so that the following relationship is met:

$$
\sin \theta_{\text {out }}=\sqrt{\frac{\varepsilon_{\text {in }}}{\varepsilon_{\text {out }}}} \sin \theta_{\text {in }}
$$

Here, $\varepsilon_{\text {in }}$ and $\varepsilon_{\text {out }}$ are the dielectric constants of the incident and emerging media respectively.

Similarly, we find: $\quad \cos \theta_{\text {in }}=\vec{n} \cdot \vec{m}$

Lastly, we give the following reference (Ouédraogo and al, 2016) related the transfer matrix method to any reader. 


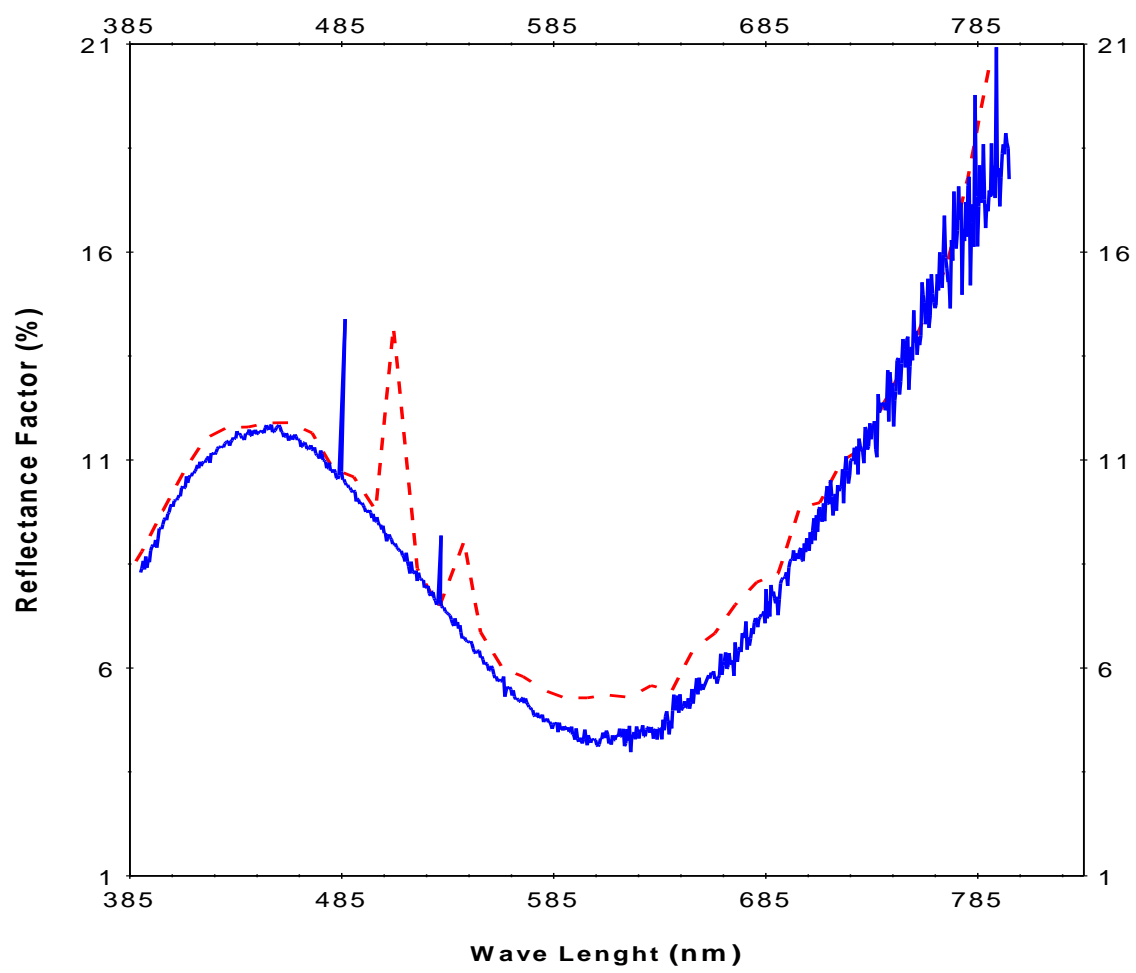

Figure 6. Reflectance Spectrum simulated and measured at normal incidence of $\theta=15^{\circ}$.

The Figure.6 shows the result obtained by modeling samples of bug elytron. This theoretical spectrum is to be compared with the experimental spectrum in Figure.2. The main peak estimated at $510.25 \mathrm{~nm}$ is located in the same spectral range as the peak of the experimental spectrum which is blue. Thus, this confirms that the multilayer observed using the SEM is responsible for the elytron color of Calidea Signata. The calculation also gives a secondary peak, with low intensity at $539.5 \mathrm{~nm}$. This corresponds to the green-bluish color. We find that the differences between the measured spectrum and the simulated one are acceptable because of the uncertainties on the angles of incidence, the average refractive indices, and especially the nonstandardization of spherical cavities.

\section{Conclusion}

In conclusion, the results of spectrophotometer measurements show a principal peak estimated at $485.5 \mathrm{~nm}$, corresponding to the blue color. A second peak, less intense was estimated at $525 \mathrm{~nm}$, and it corresponds to the green-blue color, at normal incidence of $15^{\circ}$. SEM images showed the presence of a multilayer, responsible for the blue color of the elytron of Calidea Signata bug. The curve of the surface of the elytron has a complex 
geometry leading to iridescent effect. The wavelength calculated using the Bragg mirror system gives a value of $508.25 \mathrm{~nm}$ for the first peak and 535.5 $\mathrm{nm}$ for the second peak. Numerical modeling of the experimental spectrum by the ray tracing program, combined with the transfer matrices show the first peak at $510.25 \mathrm{~nm}$ and at $539.5 \mathrm{~nm}$ for the second. This, however, corresponds corresponding to the blue and green-blue color, respectively. The curve of the multilayer of the elytron causes double reflections of rays resulting in an iridescent effect. The difference noticed in results is mainly due to the imprecision of the incidence angles at different scales. On the whole, there is good alignment between the results measured and those simulated. The multilayer is responsible for the blue reflections of the bug elytron, while the curvature is responsible for the iridescent effect.

\section{Acknowledgement}

The authors wish to thank Professor Oumarou SAVADOGO of the Laboratory of New Materials for Energy and Electrochemistry Polytechnique Montreal (Canada), for the valuable assistance given in the course of using the spectrophotometer and SEM analysis.

\section{References:}

1. Fox, L; (1976).Animal Biochromes and Structural Colors, University of California Press, Berkeley.Vukusic, P; Sambles, J. R.(2003).Photonic structures in biology.

2. Nat. J. (PP.852-855). DOI:10.1364/OE.24.012267

3. Balint, Zs; Vértesy, Z; Biro, L .P ;( 2005).Microstructures and nanostructures of high Andean Penaincisalia lycaenid butterfly scales. J. Nat. Hist (pp.2935-2952). DOI: 10.1080/00222930500140629 Berthier, S. (2007) Iridescences, the Physical Colors of Insects. Springer-Verlag, Paris, France.

4. Parker, A. (2005). Seven Deadly Colors. The Genius of Nature's Palette and How it Eluded Darwin Free Press. Simon \& Schuster London.

5. Schuh, R; and Slater, J. (1995). True Bugs of the World (Hemiptera Heteroptera). Natural History Cornell Classification. University Press, Ithaca.

6. Vigneron, J; Simonis, P; Aeillo, P ; 2010). A Reverse Color Sequence in the diffraction of white Light by the wing of the mal butterfly Pierella luna. Phys Rev E, (PP.229). DOI:10.1103/PhysRevE.82.021903 
7. ICI.(1931). International Commission on Illumination (ICI).Cambridge University Press, Proceedings.

8. Kelly, K. L.(1976).Color Universal Language and Dictionary of Names U.S. Department of Commerce, Washington DC.

9. Zi, J; Yu, X; Li, Y; Hu, X; Xu, C; Wang, X; Liu, X; Fu, R;(2003). Coloration

10. Strategies in peacock feathers. In Proc Natl Acad Sci; 100 (pp.1255612576), USA. DOI:10.1073/pnas.2133313100

11. Prendry, J; Kinnon, Mc; (2005). Calculation of photon dispersion relations.

12. Phys Rev. Lett, (PP. 69 2750-2772). DOI:10.1103/PhysRevLett.69.2772

13. Tian, B; Zheng, X; Kempa, T. J; Fang, Y; Yu, N; Yu, G; Huang, J ;( 2007).J. Nature, (PP.449-885). DOI: 10.1038/nature06181

14. Gohy, J.F; Melinte, S;(2009). J. Nano. Lett (PP. 2838). DOI: $10.1039 / \mathrm{b} 801151$

15. Huang, J; Wang, X; Wang, Z. L;(2008). J. Nanotechnology, (PP. 02560). DOI: $10.1088 / 0957-4484 / 19 / 45 / 455605$

16. Ouédraogo, I; Ouédraogo, B; Nanema, E; (2016).Structural Layer origin of the blue color reflections the wings of the Junonia Orithya Madagascarensis. European Scientific Journal (PP.147-156). DOI: 10.19044/esj.2016.v12n24p147 